\title{
Extraction-Separation Performance and Dynamic Modeling of Orion Test Vehicles with Adams Simulation: $3^{\text {rd }}$ Edition
}

\author{
Jose G. Varela ${ }^{1}$ \\ GeoControl Systems, Inc., Houston, TX, 77058 \\ Satish Reddy ${ }^{2}$ \\ Jacobs Engineering, Houston, TX, 77058 \\ Enrique Moeller ${ }^{3}$ \\ Barrios Technology, Houston, TX, 77058 \\ Keith Anderson ${ }^{4}$ \\ Orbital-ATK, Brigham City, UT 84302
}

\begin{abstract}
NASA's Orion Capsule Parachute Assembly System (CPAS) Project is now in the qualification phase of testing, and the Adams simulation has continued to evolve to model the complex dynamics experienced during the test article extraction and separation phases of flight. The ability to initiate tests near the upper altitude limit of the Orion parachute deployment envelope requires extractions from the aircraft at $35,000 \mathrm{ft}$ MSL. Engineering development phase testing of the Parachute Test Vehicle (PTV) carried by the Carriage Platform Separation System (CPSS) at altitude resulted in test support equipment hardware failures due to increased energy caused by higher true airspeeds. As a result, hardware modifications became a necessity requiring ground static testing of the textile components to be conducted and a new ground dynamic test of the extraction system to be devised. Force-displacement curves from static tests were incorporated into the Adams simulations, allowing prediction of loads, velocities and margins encountered during both flight and ground dynamic tests. The Adams simulation was then further refined by fine tuning the damping terms to match the peak loads recorded in the ground dynamic tests. The failure observed in flight testing was successfully replicated in ground testing and true safety margins of the textile components were revealed. A multi-loop energy modulator was then incorporated into the system level Adams simulation model and the effect on improving test margins be properly evaluated leading to high confidence ground verification testing of the final design solution.
\end{abstract}

\section{Nomenclature}

$\begin{array}{ll}\text { CDT } & =\text { Cluster Development Test } \\ \text { CPAS } & =\text { Capsule Parachute Assembly System } \\ \text { EFTC } & =\text { Electronic Force Transfer Coupling } \\ \text { EM } & =\text { Energy Modulator } \\ \text { EPJD } & =\text { Extraction Parachute Jettison Device } \\ \text { KIAS } & =\text { Knots Indicated Airspeed } \\ \text { LVAD } & =\text { Low Velocity Airdrop } \\ \text { MARM } & =\text { Mid-Air Release Mechanism } \\ \text { MSL } & =\text { Mean Sea Level } \\ P T V & =\text { Parachute Test Vehicle } \\ \varepsilon & =\text { Strain } \\ \text { Fmax } & =\text { Maximum breaking force of a textile line } \\ \text { Ln } & =\text { Length of a given loop number of the multi-loop energy modulator }\end{array}$

\footnotetext{
${ }^{1}$ Aerospace Engineer, Aeroscience and Flight Mechanics, 2224 Bay Area Blvd, Houston, TX, AIAA Sr. Member. ${ }^{2}$ Chief Engineer, Engineering Department, 2224 Bay Area Blvd, Houston, TX

${ }^{3}$ Aerospace Engineer, Aeroscience and Flight Mechanics, 2224 Bay Area Blvd, Houston, TX

${ }^{4}$ Aerospace Engineer, Advanced Engineering, P.O. Box 707, Brigham City, UT.
} 


\section{Introduction}

$\mathrm{T}$ he Adams simulation tool has been a cornerstone in providing the necessary analysis to set up conditions to test the corners of the Capsule Parachute Assembly System (CPAS) performance envelope. The high fidelity modeling of the extraction and separation of the mated Parachute Test Vehicle/Cradle Platform Separation System (PTV/CPSS) has been the result of incorporating data from air drop and ground testing. The first two papers in this series focused on model improvements based on drop test reconstructions; including hardware configuration changes, with the primary focus on improving pitch plane dynamics modeling. The edition focuses on the static and dynamic ground testing effort attempting to characterize and reduce the harness and reposition deployment lines loads during the backstop reposition event illustrated in Figure 1.

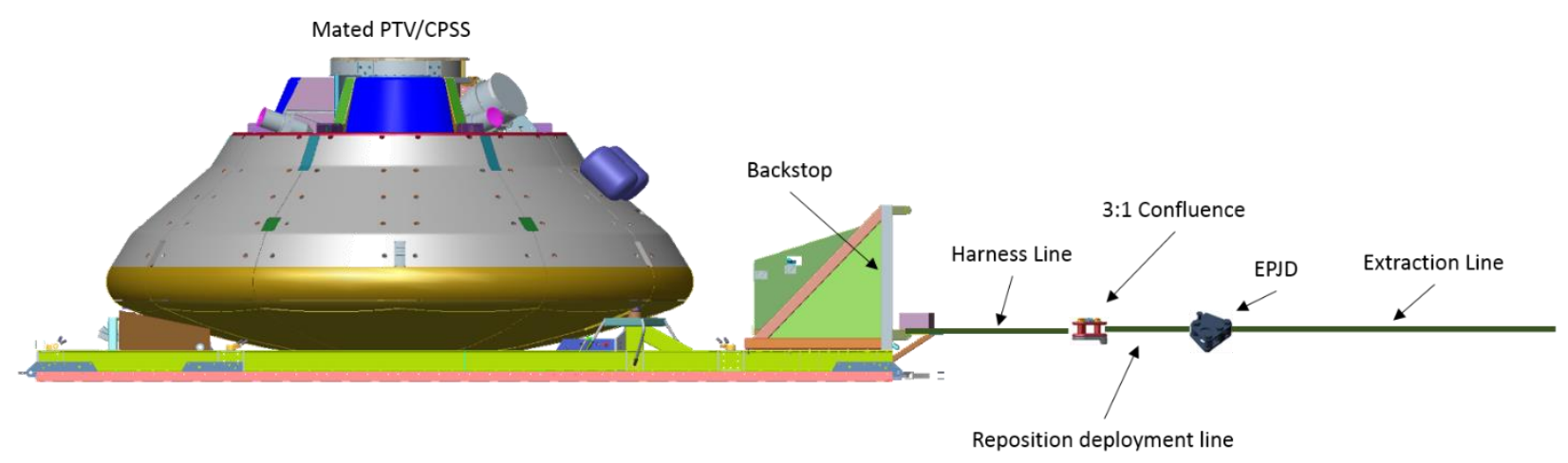

Figure 1. Load Train configuration of the reposition event of the PTV/CPSS

\section{High altitude extraction}

The desire to understand CPAS parachute performance at the upper altitude limit (35,000 ft-MSL) of the parachute deployment envelope required the ability to start the test conditions at higher altitudes than previously

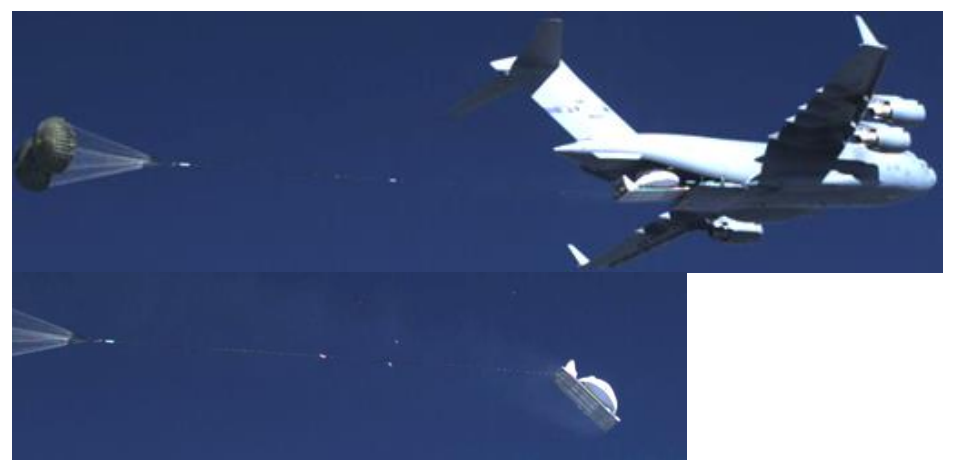

Figure 2 Chase view of PTV/CPSS extraction from C-17 tested. The first test that the CPAS project executed at 35,000 ft-MSL was Cluster Development Test (CDT)-3-11, which is seen from chase aircraft images in Figure 2. Performing the test at the higher altitude required the $\mathrm{C}-17$ carrier aircraft to fly at an airspeed of 190 Knots Indicated Air Speed (KIAS) which was a higher energy extraction than previously experienced. This resulted in two issues, the first of which was a traveling wave event that occurred during extraction parachute deployment, causing a whiplash effect when the attached EPJD was lifted up and slammed down causing damage to the aircraft deck ${ }^{1}$. This event was mitigated by securing the extraction line to the deck with ties-downs as well as modification to the extraction riser.

The second issue was discovered following post-flight inspection of hardware. Damage to the platform extraction bracket that connects the CPSS and EPJD was observed. This was attributed to the bending moment occurring between the extraction and separation events. The EPJD is free to swing upward, but a hard stop prevents it from rotating below the CPSS platform. As illustrated in Figure 3, the extraction parachutes typically align with the local velocity vector, creating a moment when the extraction line is at a negative angle of attack relative to the CPSS during the pitch-down motion. 


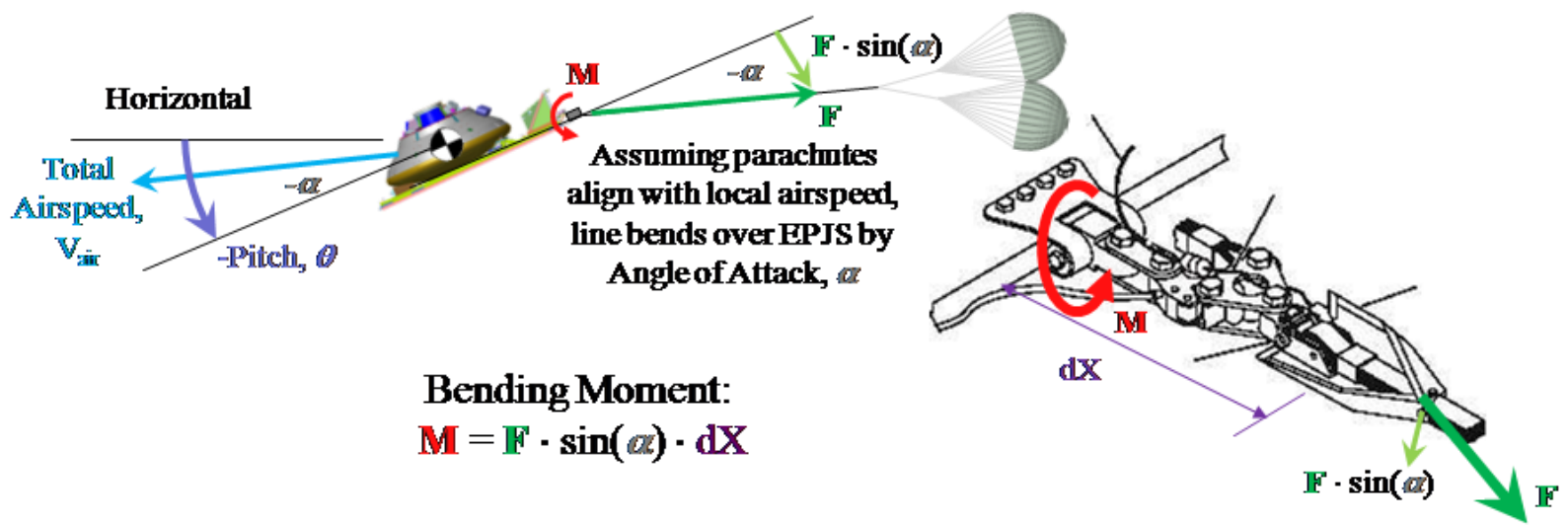

Figure 3. EPJD bending moment due to the pitch down motion of the mated PTV/CPSS

With the magnitude of the bending moment proportional to the extraction parachute force and magnitude of the angle of attack, the conditions for this test were compared to previous PTV tests in Table 1. The extraction force during CDT-3-11 was about 39\% higher than previous tests at the time of peak load (just after first motion) and the

Table 1. Extraction Conditions Contributing to Bending Moment

\begin{tabular}{|c|c|c|c|c|c|c|c|c|c|c|c|c|}
\hline $\begin{array}{c}\text { Test Name } \\
\text { or Data } \\
\text { Source } \\
\text { Number }\end{array}$ & $\begin{array}{l}\text { Altitude } \\
\text { (ft MSL) }\end{array}$ & $\begin{array}{c}\text { Knots } \\
\text { Calibrated } \\
\text { Airspeed } \\
\text { (KCAS) }\end{array}$ & $\begin{array}{c}\text { Knots True } \\
\text { Airspeed } \\
\text { (KTAS) }\end{array}$ & $\begin{array}{c}\text { Extracted } \\
\text { Payload } \\
\text { Weight } \\
\text { (lbm) }\end{array}$ & $\begin{array}{c}\text { Number of } \\
\text { Extraction } \\
\text { Parachutes, } \\
\mathbf{N}_{\mathbf{c}}\end{array}$ & $\begin{array}{c}\text { Reefing } \\
\text { Ratio }(\%)\end{array}$ & $\begin{array}{c}\text { Extraction } \\
\text { Drag Area } \\
\text { per canopy, } \\
C_{D} S\left(f^{2} t^{2}\right)\end{array}$ & $\begin{array}{c}\text { Mean Drag } \\
\text { Coefficient } \\
\text { per } \\
\text { canopy, } C_{D}\end{array}$ & $\begin{array}{c}\text { Peak } \\
\text { Extraction } \\
\text { Force (lbf) }\end{array}$ & $\begin{array}{l}\text { Extraction } \\
\text { Force at } \\
\text { Max. Pitch } \\
\text { (lbf) }\end{array}$ & $\begin{array}{c}\text { Max. } \\
\text { Extraction } \\
\text { Pitch Angle } \\
\text { (deg) }\end{array}$ & $\begin{array}{c}\text { Max. } \\
\text { Extraction } \\
\text { Angle of } \\
\text { Attack } \\
\text { (deg) }\end{array}$ \\
\hline CDT-3-3 & 25,249 & 145.9 & 215.1 & 31,338 & 2 & Unreefed & 290.8 & 0.472 & 37,360 & 20,550 & -42.7 & -31.9 \\
\hline CDT-3-5 & 25,079 & 142.8 & 212.6 & 31,901 & 2 & Unreefed & 302.8 & 0.492 & 38,010 & 24,100 & -32.0 & -20.0 \\
\hline CDT-3-7 & 24,990 & 144.2 & 211.3 & 31,412 & 2 & Unreefed & 293.0 & 0.476 & 38,800 & 20,210 & -44.3 & -32.5 \\
\hline CDT-3-9 & 24,988 & 148.2 & 219.8 & 31,412 & 2 & Unreefed & 312.7 & 0.508 & 39,720 & 23,000 & -44.1 & -33.7 \\
\hline CDT-3-11 & 35,264 & 190.4 & 329.3 & 32,272 & 2 & $54 \%$ & 195.5 & 0.317 & 51,080 & 32,820 & -44.9 & -38.4 \\
\hline
\end{tabular}

force was about $28 \%$ higher than previous tests during the maximum pitch-down event. The angle of attack also had the largest magnitude yet experienced.

The sequence of images acquired during the test shown in Figure 4 , further illustrate the result of a delayed load transfer of a high energy extraction event. Beginning at the top left image it is clear that the pitch down of the mated PTV/CPSS begins. The top right and bottom left images show the progression of the pitching moment until the damage finally occurs as seen in the bottom right image.

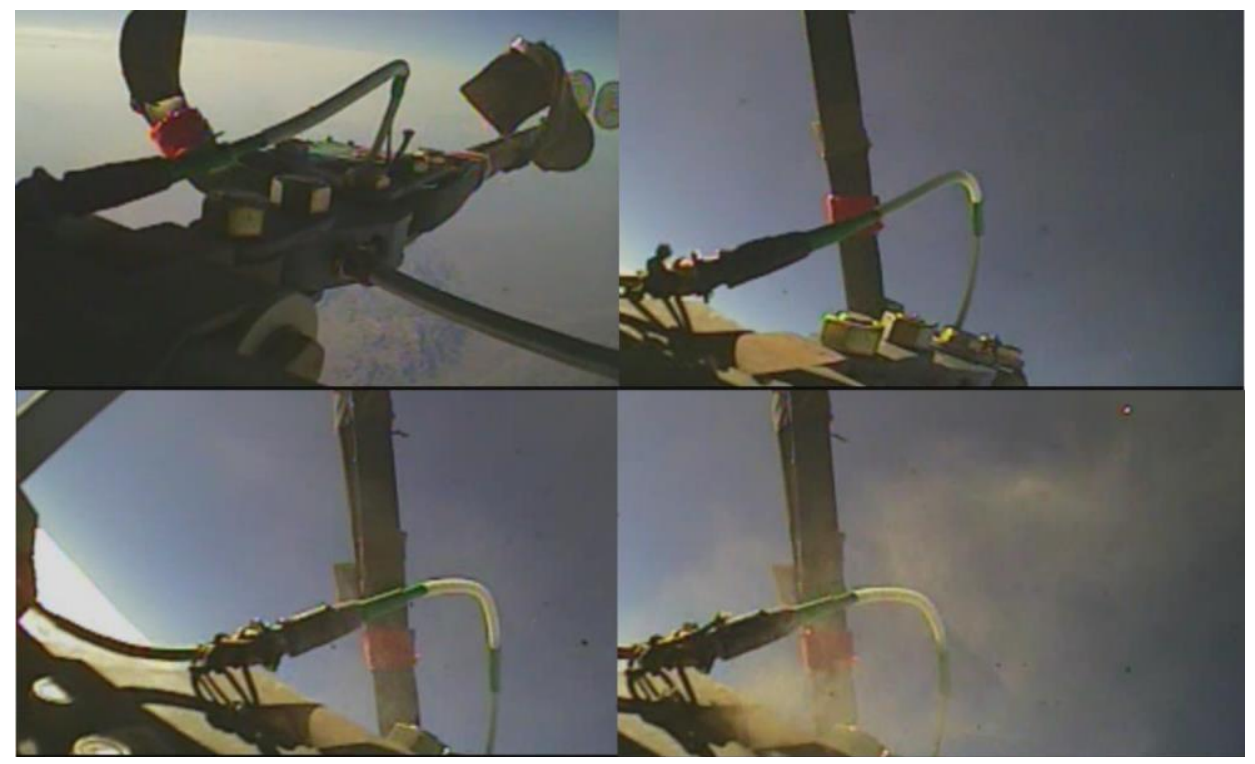

Figure 4. Concept of operations for the CPSS following the extraction and separation events

3

American Institute of Aeronautics and Astronautics 


\section{Backstop reposition mitigation strategy}

Determining a strategy for mitigating the risk associated with the bending moment damage encountered during the extraction and separation phase of CDT-3-11 became a top priority for the project. Without a fix, testing from the high altitude was unlikely, limiting the ability to test the parachute performance. As seen in Figure 5, following extraction and separation of the PTV from the CPSS, the CPSS descended under the extraction parachutes delaying the load transfer from the EPJD to the backstop at a predetermined time from ramp clear. Repositioning to the backstop harness line places the CPSS into a stable aerodynamic attitude until the recovery main parachutes are static line deployed. This previous test technique provided the best solution for smart separation as well as provided margins for the hardware and textiles located along the load train from the extraction line to the CPSS backstop.

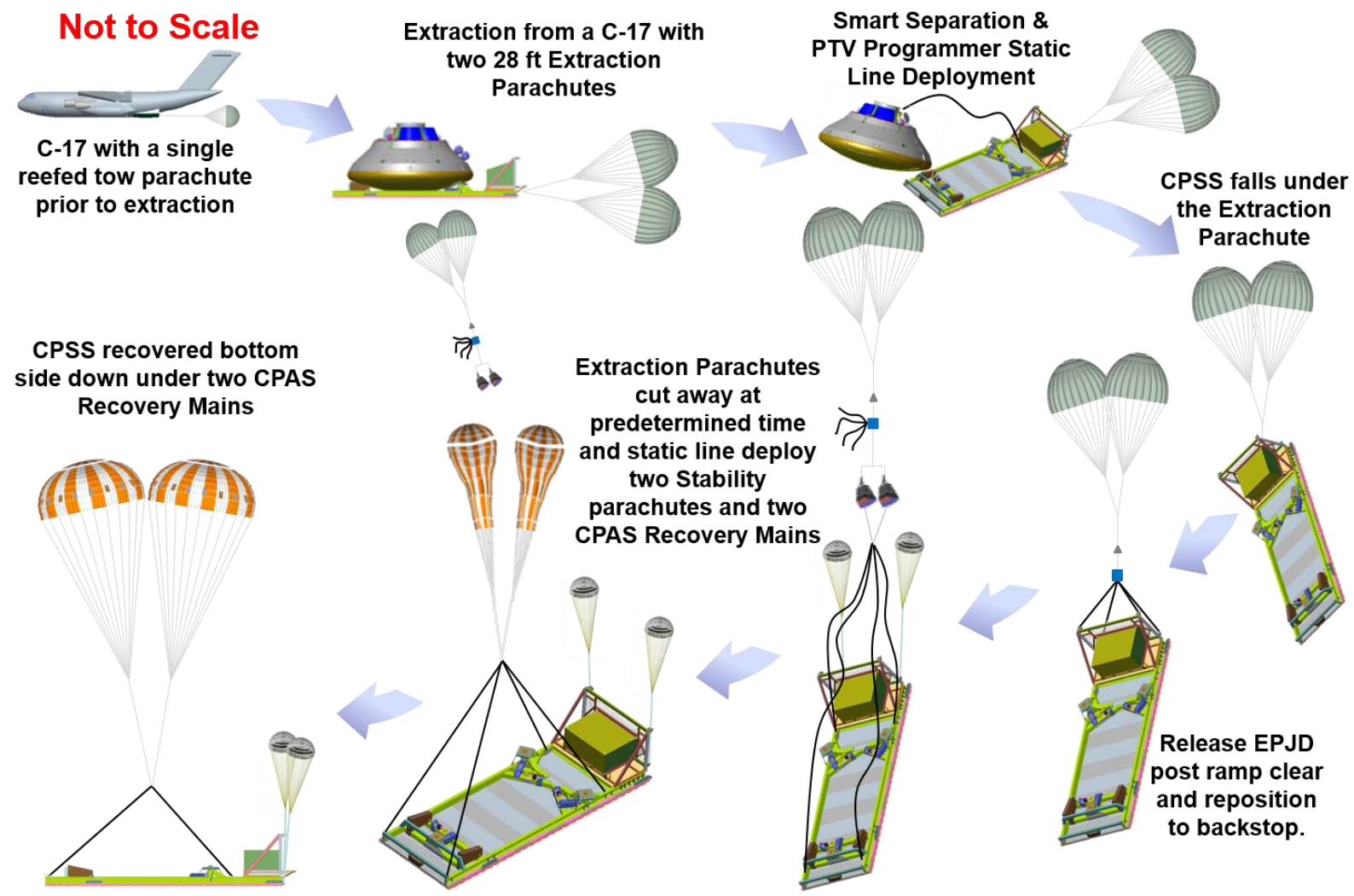

Figure 5. Concept of operations for the CPSS following the extraction and separation events

The recommended change; which turned out to be the simplest to implement yet challenging to refine, was to eliminate the delayed load transfer from the bottom of the CPSS/EPJD attach location to the CPSS backstop and allowing it to occur at ramp clear (standard LVAD technique), eliminating the bending moment damage.

Determining the effect of the modified test technique required a series of model changes. This included deconstructing the original backstop model and rebuilding it to coincide with the correct dimensions and harness attach locations. The backstop harness lines were also added and initially modeled as a simple spring mass-damper system with the damping terms derived from extraction load profiles inferred from accelerometer data. An initial attempt to model the hardware between the backstop harness line and the extraction was attempted, however, due to the complexity of incorporating objects with mass while maintaining simulation stability, it was not achieved until after the completion of CDT-3-10 (first test with the modified backstop reposition test technique) ${ }^{2}$.

A feasibility study determined the extent to which the pitch plane motion would be affected by repositioning to the CPSS backstop at ramp clear. This was critical, as changing the load path from the bottom of the CPSS to its backstop attach locations had potential to affect the smart separation window, as shown in Figure 6. Performing the 
backstop reposition event at ramp clear with the top and bottom harness line lengths in use to that point in the test program (dashed blue trace) or making them of equal length (solid red trace) would severely diminish the effectiveness of the smart separation algorithm. The only option feasible was to modify the lengths such that the lower line was loaded up through the separation event (solid green trace). This simplified model did not include the reposition deployment line connecting the EPJD to the then Mid-Air release Mechanism (MARM), and the load predictions for the segment were therefore inferred from the simulated extraction line loads. It wasn't until after completion of the CDT-3-10 test and subsequent reconstruction with a higher fidelity model that a better understanding of the actual load profile was obtained ${ }^{2}$.

\section{Characterization of Kevlar and Nylon lines}

Providing load estimates associated with the extraction phase of flight that are as close to observed values as possible has been an ongoing evolution for the CPAS analysis team. The initial model contained only the extraction line component, modeled as a simple linear spring damper system ${ }^{2}$. This approach was used until the successful execution of CDT-3-10. It took a subsequent reconstruction of that test followed by a ground test campaign to better understand the behavior of the textiles being utilized.

All ground testing was conducted and Holloway Houston Inc. Initial testing only consisted of static pull tests of $20 \mathrm{~K}$ Kevlar and type 26 nylon. When possible, the lengths of the lines were kept identical to the flight configuration. The exception was $140-\mathrm{ft}$ extraction line. The tests were conducted at the fastest pull rate available (two inches per second), with deflection and force data collected. The initial round of testing loaded the material to 60,000 lbf; after a pause it was unloaded. The cycle was repeated three times for each test. The purpose was to understand the extent to which the material stiffness changed, as well as characterize the force deflection of each line with multiple loading and unloading events. The final round of testing added a fourth pull that loaded the material to failure.

Two test samples are shown in Figure 6 where the top co-plot shows the loading and unloading curves for the first two loading events (blue solid trace represents the first loading event with the orange solid trace the second pull) for a 20k Kevlar, 6-loop Mobius test sample. The bottom plot illustrates similar information for a type 26 nylon, 8 -loop Mobius test sample. Prior to the static testing, the amount of information to characterize the various harness lines
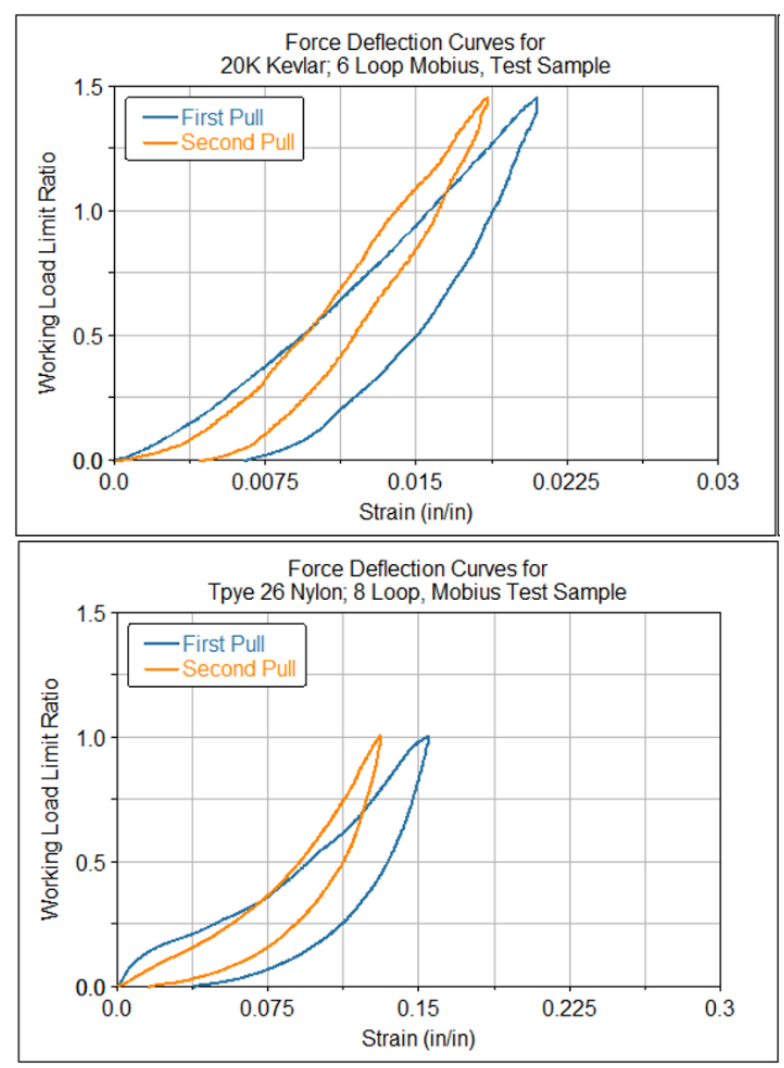

Figure 6. Force deflection curves for $20 \mathrm{~K}$ Kevlar and type 26 nylon lines implemented within the load train used a linear stiffness coefficient and maximum elongation under strain $^{2}$.The results of the testing quantified the difference between Kevlar and nylon, as the force deflection curve for Kevlar shows a much steeper increase in stiffness with increasing load. The difference in the area under the curve between the loading and unloading events represent the strain energy in each line. The data acquired was used to derive fourth-order polynomial curve fits for a given material type and line length for implementation into the Adams model.

The increased understanding obtained from the static line testing, as well as modifications made to the confluence hardware, led to the CDT-3-16 (extraction at $35,000 \mathrm{ft}$ ) analysis conclusions that there was minimal risk to the test vehicle during the backstop reposition phase of flight. However, post-flight inspection showed that, although the Kevlar reposition deployment line did not completely fail (and the test was successful), it had sustained significant damage to the Kevlar reposition deployment line, as seen in Figure 7. 


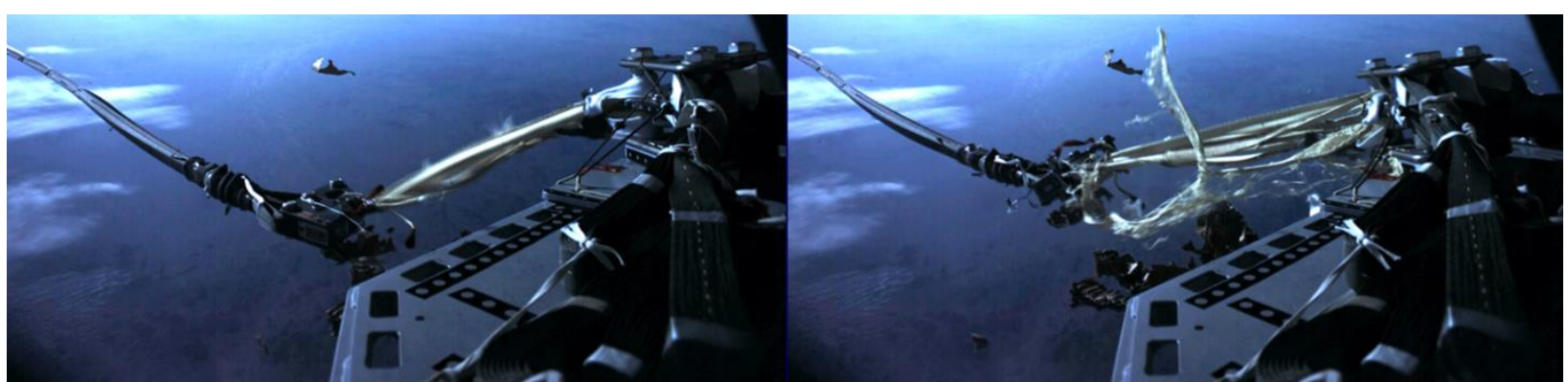

Figure 7. CDT-3-16 reposition deployment line damage

The post-test investigation revealed that the Kevlar reposition deployment line failed due to asymmetric and high loading. The line was attached to the confluence fitting in a split fashion in which four plies looped around the upper pin and four plies looped around the lower pin as illustrated in Figure 8. As a result, the loading would have made Kevlar fibers in the upper band stretch much more than under symmetric loading. Kevlar quickly becomes stiffer as it is stretched, leading to significantly higher peak loads. When the line was pulled asymmetrically, it is very likely that only one of four plies took all the load, causing its failure.

As a result of the failure, an all-nylon configuration was recommended with additional ground testing to validate modeling before another PTV/CPSS would proceed at an extraction altitude of 35,000 ft-MSL.

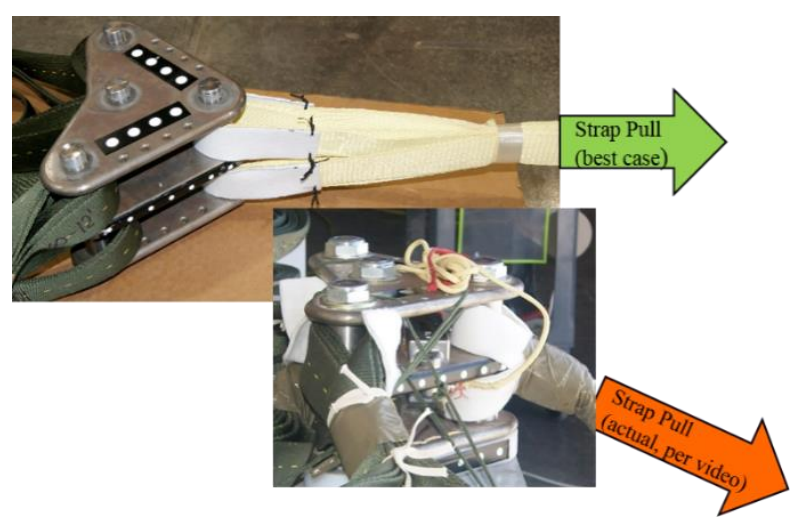

Figure 8. Orientation of 6:2 confluence as rigged for CDT-3-16

This would effectively eliminate the chance of a stress concentration in the line from occurring immediately as the confluence is pulled off the shelf. A mass emulator for the EPJD was also employed, which was a modified EFTC.

In order to validate ground test configuration and have applicability for a flight test, the configuration flown on CDT-3-16 was conducted first. The test set-up performed is illustrated in Figure 10.

\section{Dynamic ground testing}

While the static line testing of the various textile lines characterized both $20 \mathrm{~K}$ Kevlar and Type 26 nylon, the necessity to fully comprehend behavior in a dynamic loading event became paramount. Before any testing was executed, analysis was performed completed to determine if the amount of energy stored in the 140 foot extraction line would be sufficient to emulate the in-flight loading sequence. Additionally, changes to the confluence were made, reducing its total mass by about half to reduce the reposition deployment line peak load due to the momentum of the confluence. Modifications were made to the shelf on which the confluence is mounted, as seen in Figure 9, so that the line of action during the deployment event was parallel to the mounting surface.

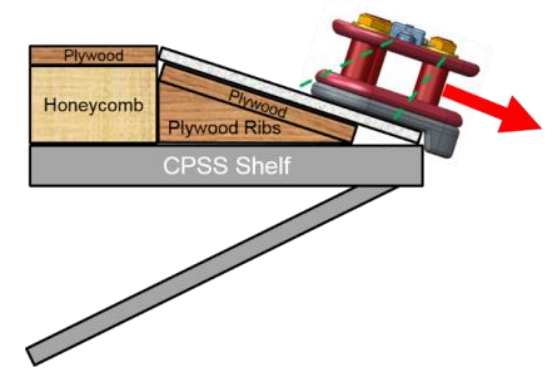

Figure 9. Modified confluence and shelf 


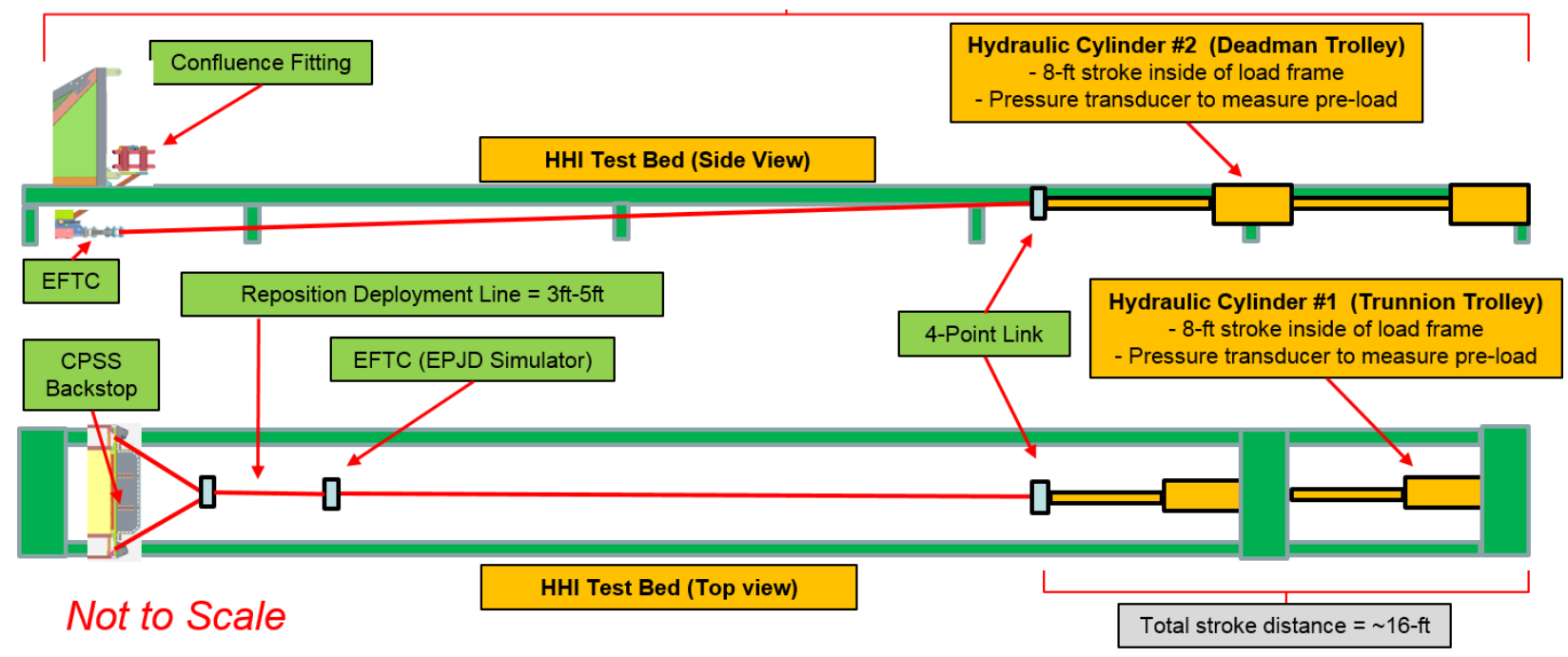

Figure 10. Illustration of the dynamic test configuration

The test bed consisted of a pair of rails with predrilled holes placed at various increments for securing the deadman and trunnion trollies, both of which have a maximum piston stroke of eight feet at the required distances. The CPSS backstop was secured to the opposite end of the test bed. The 140-foot extraction line was attached to the EFTC (EPJD emulator) at the same end, with the other end connected to a four-point link. The four-point link was then attached to the deadman trolley, which in turn was secured to the trunnion trolley with a load cell between them. In order to increase the load in the extraction line, the fully extended hydraulic arm of the secured trunnion trolley would pull the deadman trolley until the full stroke was used. Then the deadman trolley would be secured to the rail, so that the hydraulic arm of the trunnion trolley could be fully extended, after which the process was repeated. This occurred three times, followed by the fourth and last loading event performed by the deadman trolley. The force and deflection of the extraction line were recorded during this event and curves were generated to replace those previously derived from static pull tests. Once the load in the line was stable, the EFTC actuator arm was manually engaged, releasing it at the attach location to initiate the reposition sequence.

Five tests were completed during this session. The first two used the CDT-3-16 configuration which consisted of Type 26 nylon backstop harness lines and a $20 \mathrm{~K}$ Kevlar reposition deployment line. The first test failed with the reposition deployment line completely failing and sending the EFTC flying into the deadman trolley, as seen in Figure 11. Inspection of the hardware showed a sharp corner existed where the reposition deployment line attached to the EFTC. This was removed before the next test commenced, which was successful with the load estimates for the test (solid red trace) co-plotted with both ground and flight test simulation data (solid blue and green traces) in Figure 12. The final three tests were set-up as an all nylon configuration with the modified confluence. Test three loaded the extraction line to the expected, nominal value consistent with those flown in 35,000 ft-MSL tests. Tests four and five were overload cases with the extraction line loaded to 1.1 and 1.5 times the nominal value of test three,

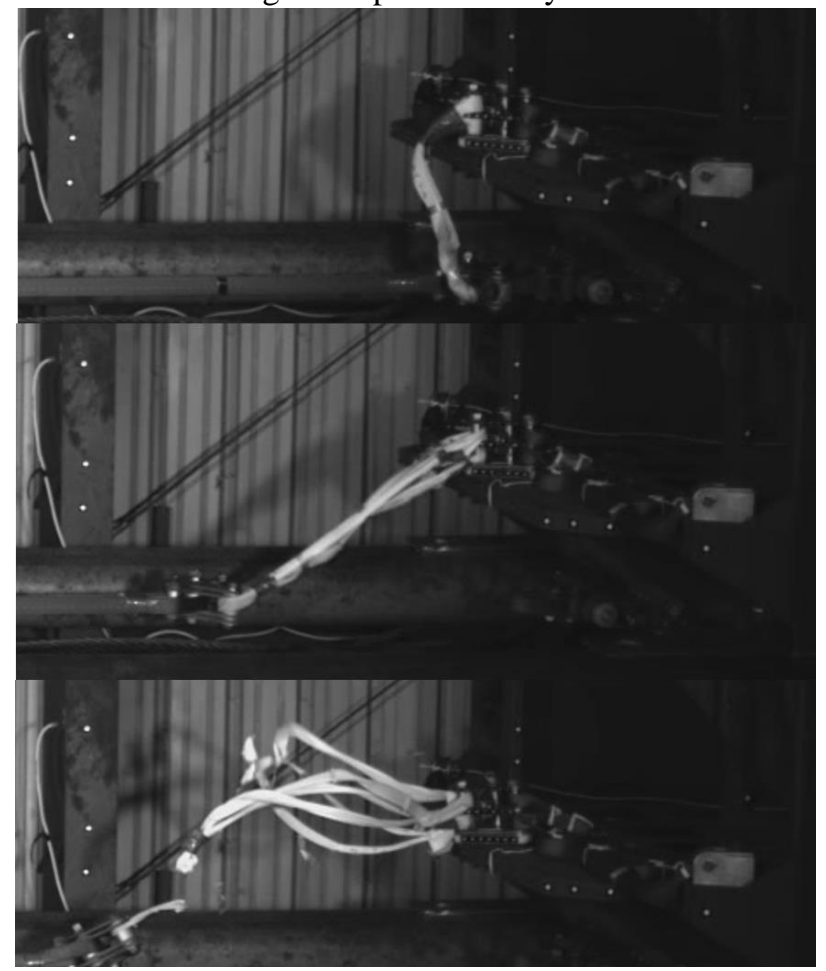

Figure 11. Dynamic test 1; Kevlar reposition deployment line

American Institute of Aeronautics and Astronautics 
respectively. Only test three was successful, with tests four and five experiencing reposition deployment line failures.

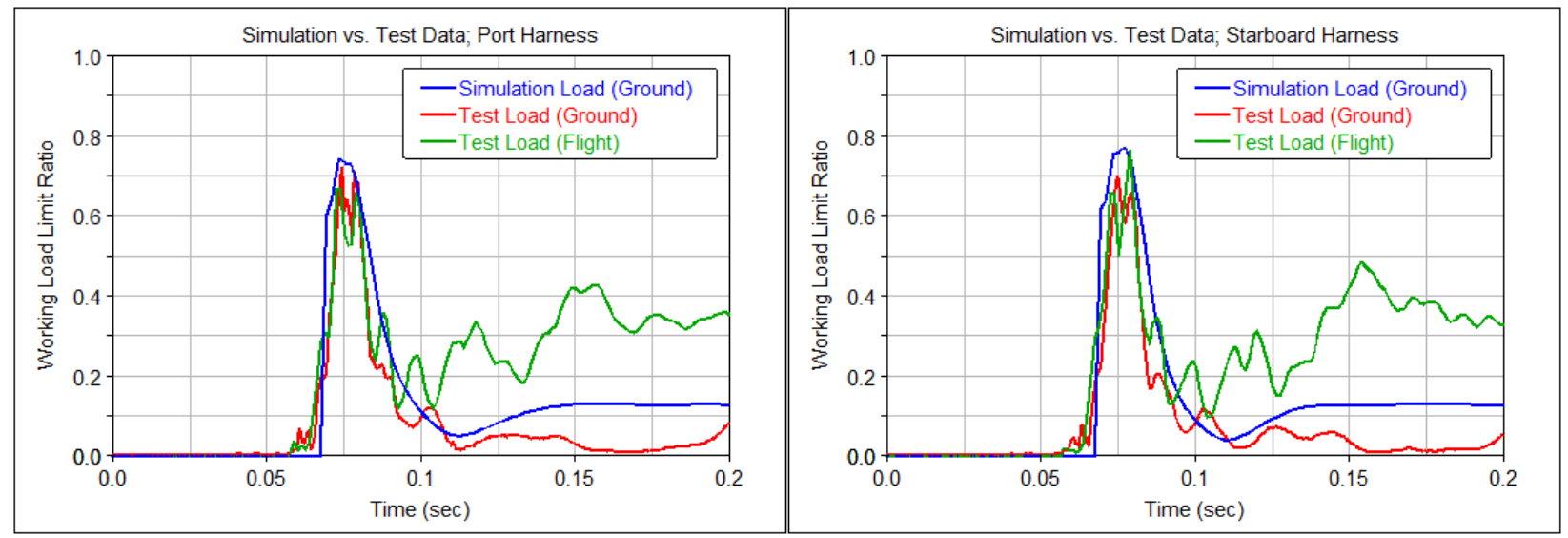

Figure 12. Dynamic test 2 backstop harness line load profiles; Kevlar reposition deployment line

\section{Multi-loop energy modulator}

Following the initial dynamic ground testing of the backstop reposition event, determination of a method to reduce loads without significant hardware modification became a priority. Initial strategies focused on techniques that were achievable with limited changes to the test support equipment. Emphasis was placed on a design to reduce the amount of energy available in the extraction line at the beginning of the reposition event. Options considered (in order of increasing difficulty) included moving the payload forward from the current location in the C-17, delaying the reposition event a short time after ramp clear, increasing the length of the backstop harness lines, reducing the extraction parachute drag area and implementing energy modulation between the backstop and the confluence. These strategies each provided some reduction in the estimated peak reposition deployment line load individually, and the combination of all of them provided the largest reduction. However, the only options that appeared to be adaptable for ground testing were increasing the length of the backstop harness lines and implementing an energy modulator. Potential for increasing the harness line lengths beyond the current configuration proved to be limited on the ground, as the simulated test cases indicated there would be insufficient strain energy in the extraction line to fully load the system at the start of the test. Therefore, the only option with a reasonable chance was the installation of an energy modulator between the CPSS and the confluence.

The proposed energy modulator was non-standard, as instead of reducing a load through breaking of stitching, load reduction would be achieved by breaking a sacrificial multi-loop line. Each of the loops would be slightly longer than the previous loop in order to allow for breaking to occur sequentially. The material selected was Type 8 nylon with a breaking strength of 4,000 lbf, or 8,000 lbf for a single loop, with an estimated strain at failure of $25 \%$. The configuration selected was a five loop modulator with an inner loop length of 48 inches and an outer loop length of 60 inches as depicted in figure 17.

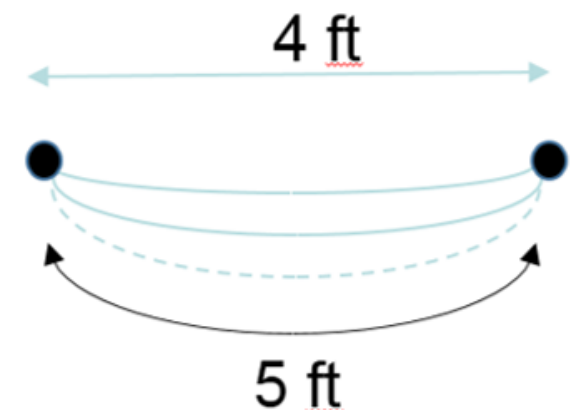

Figure 13. Conceptual view of the multi-loop energy modulator 
Estimating the force deflection of the multi-loop energy modulator begins with the assumption that a quadratic equation mathematically describes the behavior with loading.

$$
F(\varepsilon)=A \varepsilon^{2}+B \varepsilon
$$

Assuming that the slope is zero when strain is zero and substituting the maximum strain and load values.

$$
A=\frac{F_{\max }}{\varepsilon_{\max }^{2}}
$$

The function for the strain range is greater than equal to zero and less than to equal the maximum strain, becomes.

$$
\mathrm{F}(\varepsilon):=\mid \begin{aligned}
& \left(\frac{\mathrm{Fmax}}{\operatorname{cmax}^{2}} \cdot \varepsilon^{2}\right) \text { if } 0 \leq \varepsilon \leq \max \\
& 0 \text { otherwise }
\end{aligned}
$$

With this function a theoretical force deflection curve for a single loop is generated and illustrated in Figure 14.

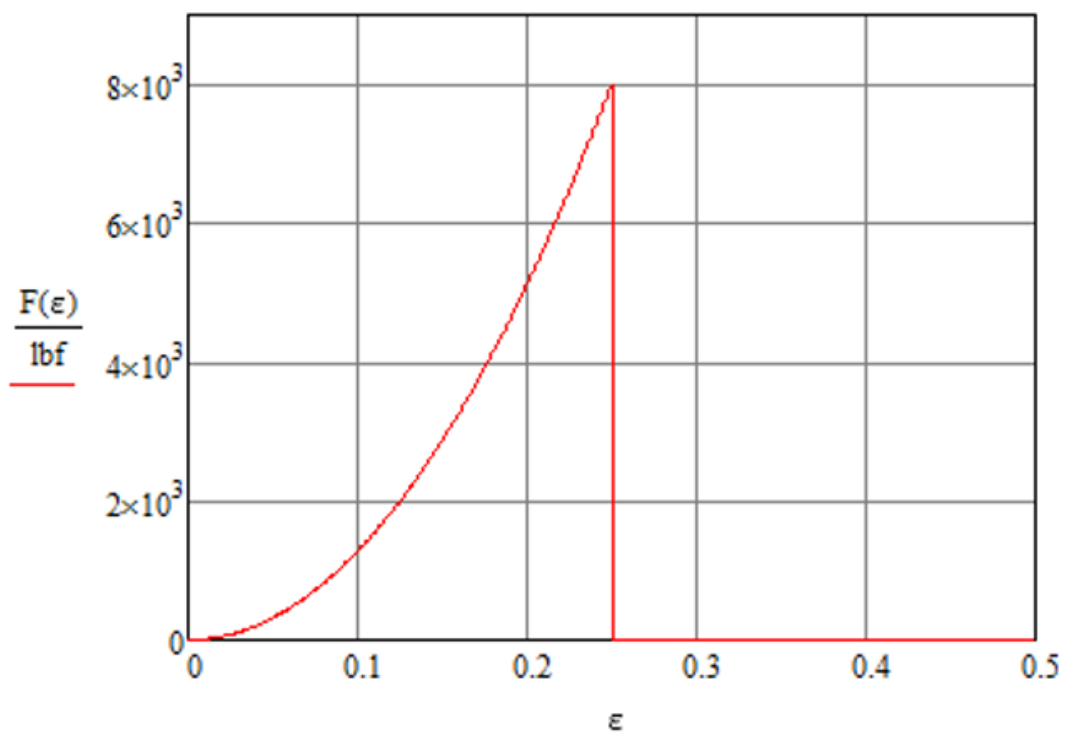

Figure 14. Theoretical force deflection curve for a single loop type 8 nylon line

For sequentially loaded individual loops, the equation (3) becomes (with the plot shown in Figure

$$
\underset{M}{\mathrm{P}}(\mathrm{x}, \mathrm{n}):=\mid \begin{aligned}
& {\left[\frac{\mathrm{Fmax}}{\varepsilon \max ^{2}} \cdot\left(\frac{\mathrm{x}-\Delta_{\mathrm{n}}}{\mathrm{L}_{\mathrm{n}}}\right)^{2}\right] \text { if } 0 \leq \mathrm{x}-\Delta_{\mathrm{n}} \leq \operatorname{\varepsilon max} \cdot \mathrm{L}_{\mathrm{n}}} \\
& 0 \text { otherwise }
\end{aligned}
$$




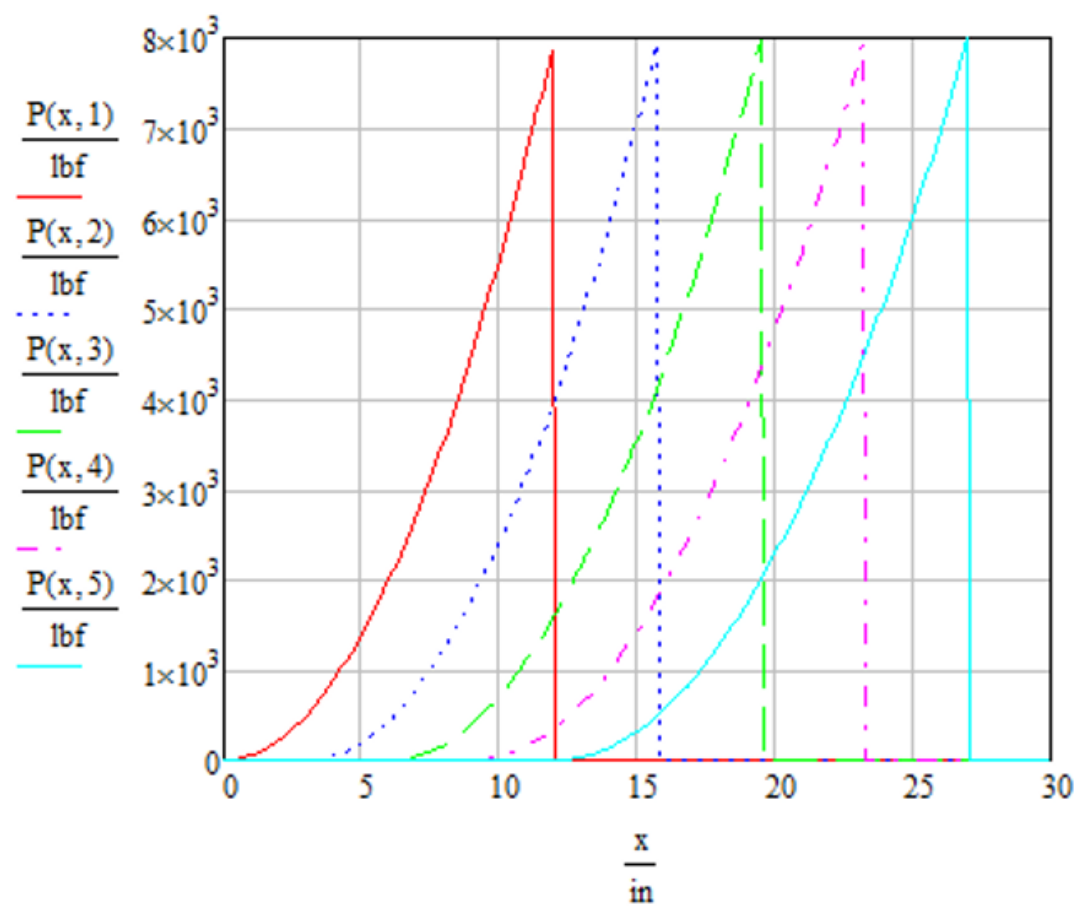

Figure 15. Theoretical force deflection curves for a five loop energy modulator

Combining the individual force deflection curves, the resultant is expressed in equation (5) and the associated curve plotted in Figure 16.

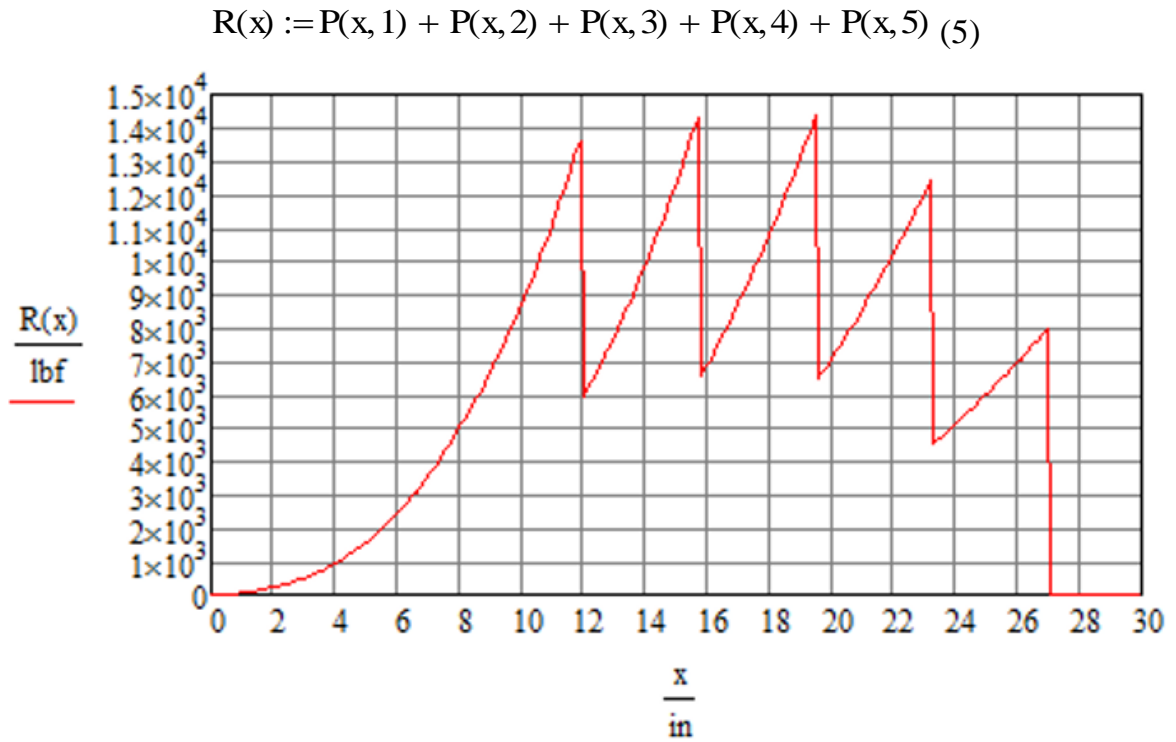

Figure 16. Resultant theoretical force deflection curve of a five loop energy modulator

The theoretical multi-loop energy modulator was implemented into the Adams simulation in order to estimate the effectiveness of this risk mitigation strategy. Figure 17 illustrates the deployment sequence in a series of images. Images 1 and 2 show the release of the EPJD, which in turn deploys the confluence via the reposition deployment line, with no load in the energy modulator (represented by the green point masses) until it reaches the distance of the inner loop length. Images 3 through 7 show that once an individual loop reaches its peak load, it will fail and the next loop will load until it reaches its peak value. Image 8 shows the full extension of the system with the multi-loop energy modulator has performed its function. 


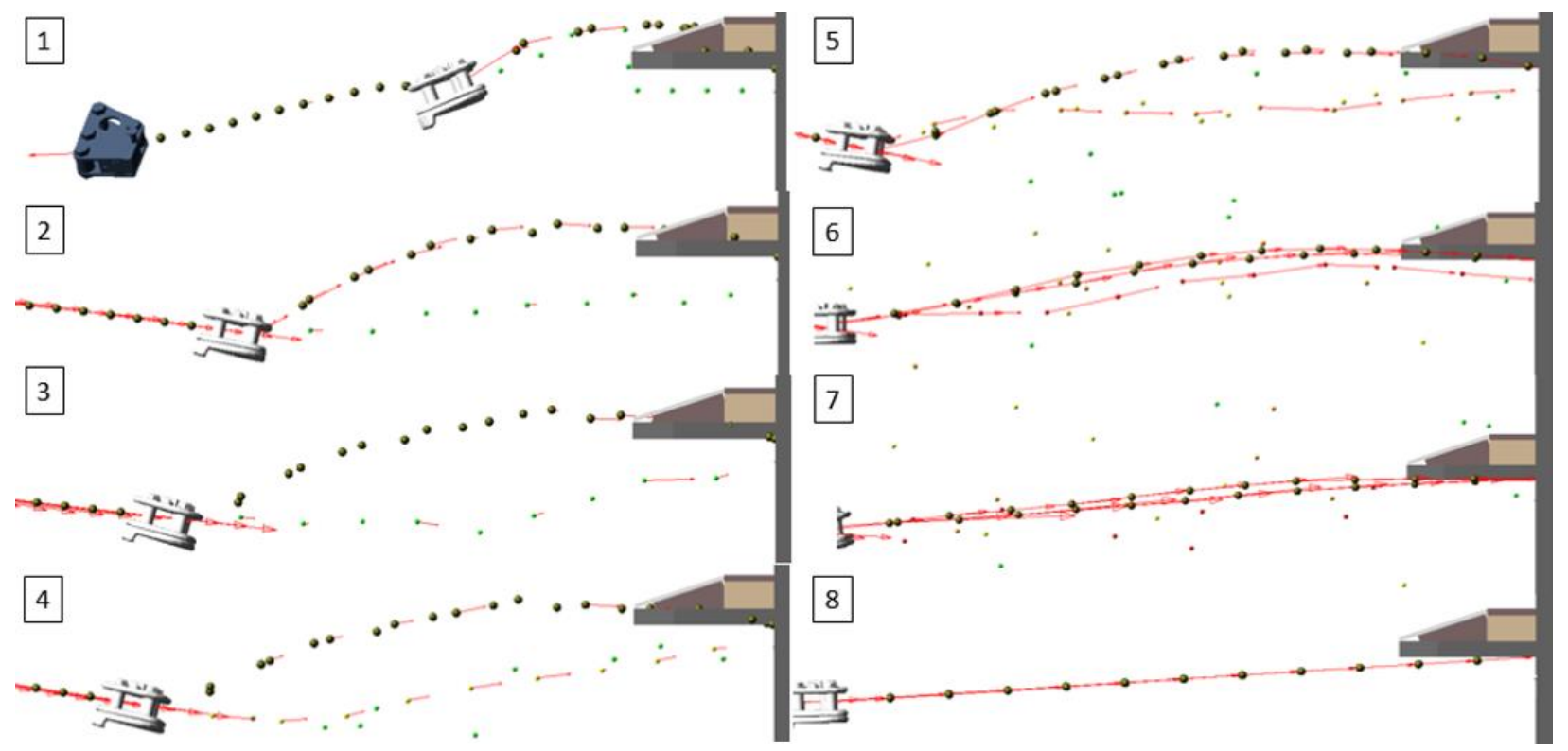

Figure 17. Deployment sequence of the multi-loop energy modulator in the Adams simulation

Verification of the multi-loop energy modulator force deflection curve, was sought before dynamic ground testing was performed. A set of six multi-loop energy modulators were fabricated for static testing. Only five of the six tests were successful in acquiring the necessary data, due the string pot inadvertently detaching from the test stand. The force deflection curves and resulting work performed for tests two and six are co-plotted in Figure 18. The primary behavior observed in the tests was that the two inner loops adhered to one another, increasing the amount of force required to make the first break. In addition, while there was an inconsistency between tests in the pattern of the force deflection curves, the amount of work done (strain energy) in breaking each test case had a standard deviation of only $5 \%$.

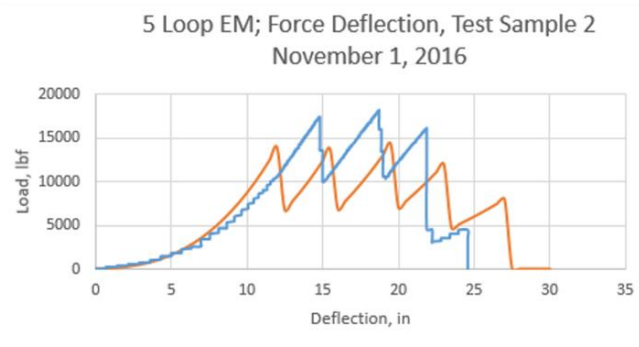

- Theoretical - Test Sample 2

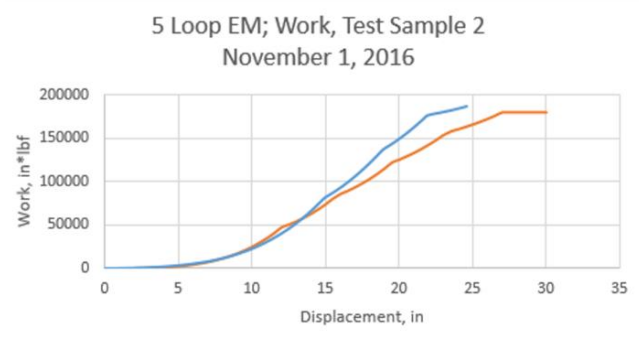

- Theoretical —Test Sample 2

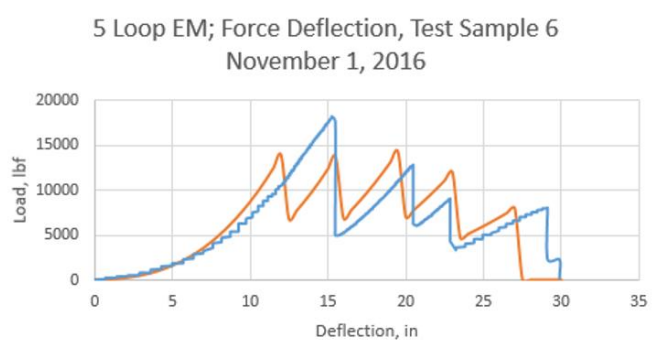

-Theoretical L Series 1

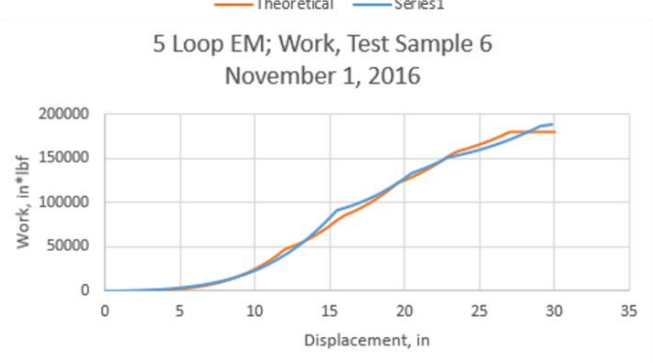

—Theoretical —TestSample 6

Figure 18. Force deflection and energy curves for the multi-loop energy modulator static test numbers 2 and 6

The test proved that the energy modulator would behave similar to the derived theoretical force deflection curve. This is shown in Figure 19, where the reposition deployment line as well as the port and starboard harness line working load limit (WLL) ratios are compared to a case with no energy modulator (red trace), use of the theoretical 
energy modulator (blue trace) and the results of test samples two and six (green and dark blue traces respectively). This analysis indicated that a $15 \%$ reduction in the peak reposition deployment line could be achieved by implementing the multi-loop energy modulator.
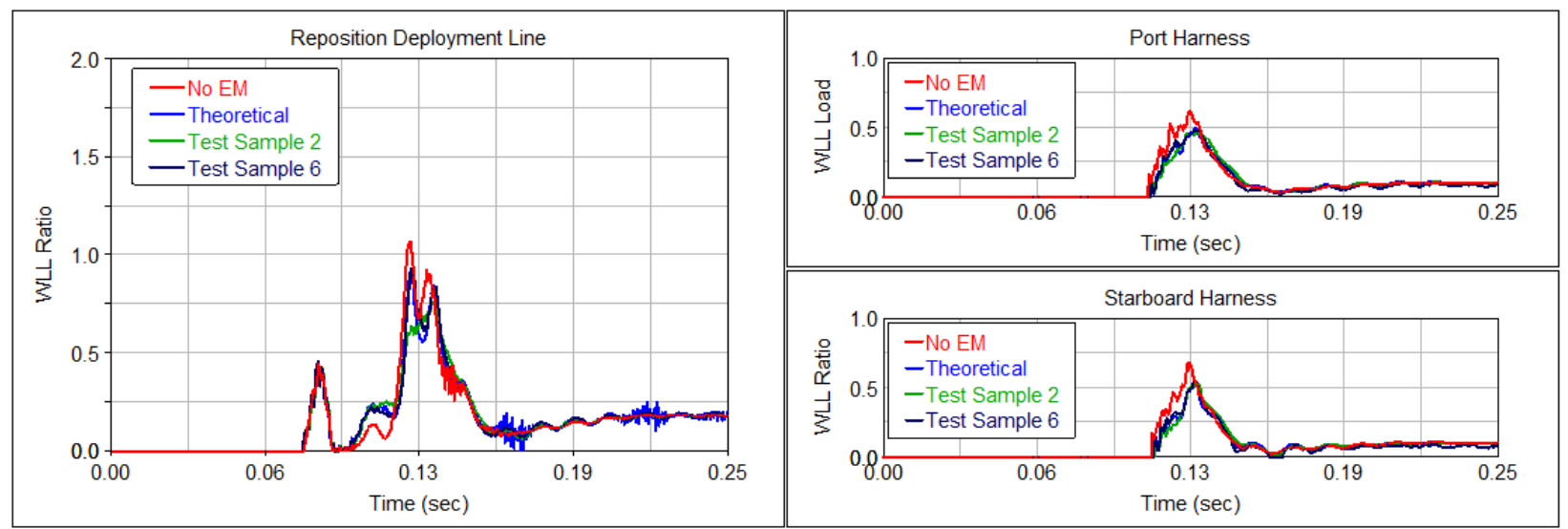

Figure 19. Reposition deployment and harness line working load limit ratio comparing the no energy modulator case to the derived energy modulator and two of the static line test cases

\section{Dynamic ground testing with a multi-loop energy modulator}

With analysis indicating that the multi-loop energy modulator would provide the necessary load reduction mitigation strategy, dynamic ground tests were performed. The initial recommendation was to run six cases, three with the nominal extraction line load and three with a factor of 1.1 applied to the same load as overload test cases, which was the three sigma value for the extraction line load of the Monte Carlo analysis. The tests were executed in the same manner as previously discussed.

Test 1, run with the nominal extraction line load, was successful. Tests 2 through 4 were the three sigma extraction line load cases all of which performed flawlessly. Following completion of test 4 , the team modified the test strategy to run tests 5 and 6 with a factor of 1.2 and an additional test with a factor of 1.25 . All test were successful. Ten out of twelve derived force deflection curves are plotted in Figure 20. The first set of dynamic tests are shown as solid curves and denoted with the test number and no EM, where the remaining tests (dashed lines) indicate the tests with the EM. The two tests that are not plotted are test two and seven from the tests with the EM, as a data acquisition error occurred that prevented derivation of a force deflection curves. 


\section{Derived Force Deflection Curves \\ $140 \mathrm{ft}$ Extraction Line \\ Type 26 Nylon; 6-Loop}

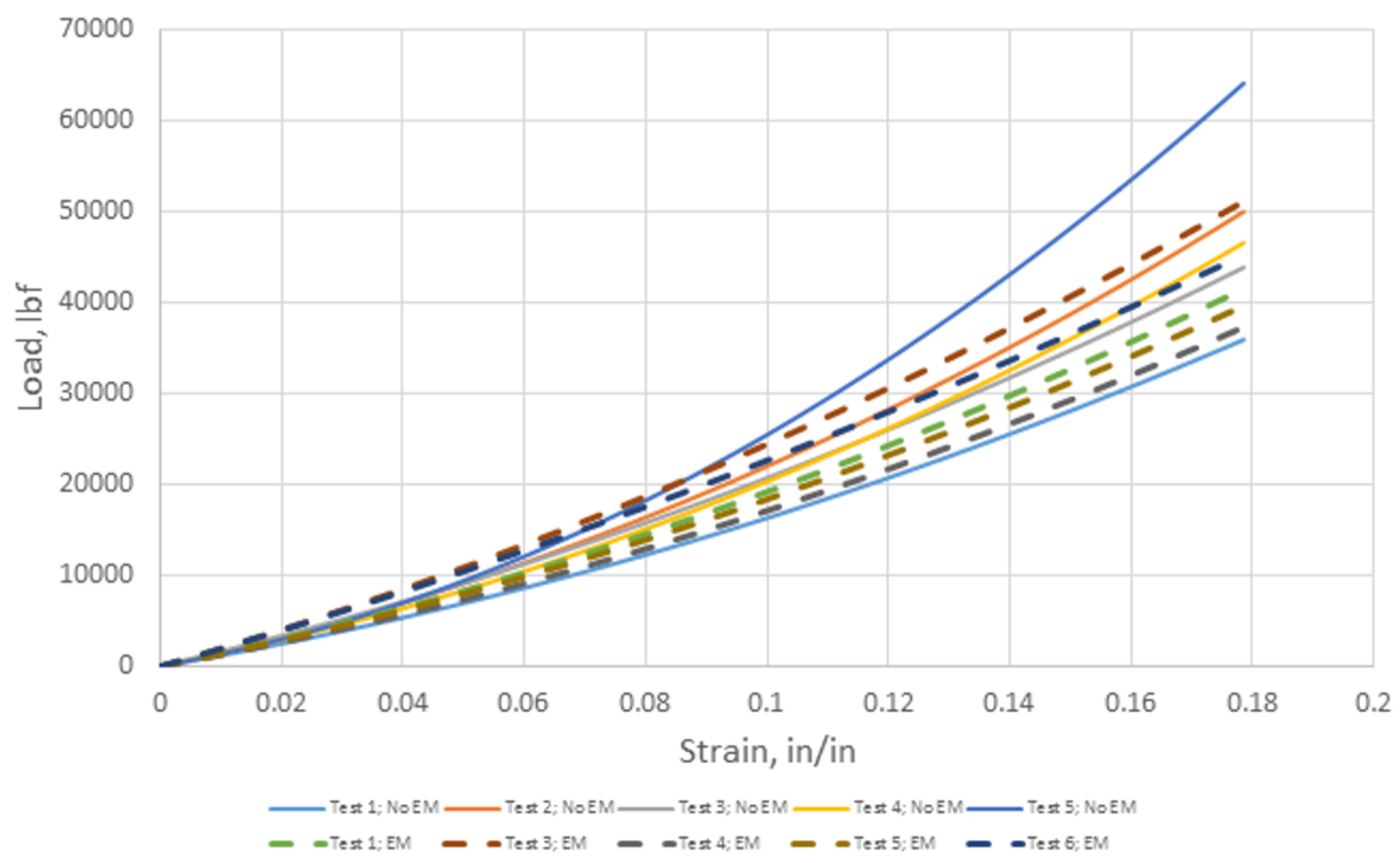

Figure 20. Derived force deflection curves for a 140 foot, 6-loop type 26 nylon extraction line

Co-plots of the predicted values for the reposition deployment and backstop harness line are show in Figure 19. The predicted peak reposition deployment line value was roughly $2 \%$ lower than tested. The predicted port and starboard backstop line value came in at $17 \%$ higher than as measured.
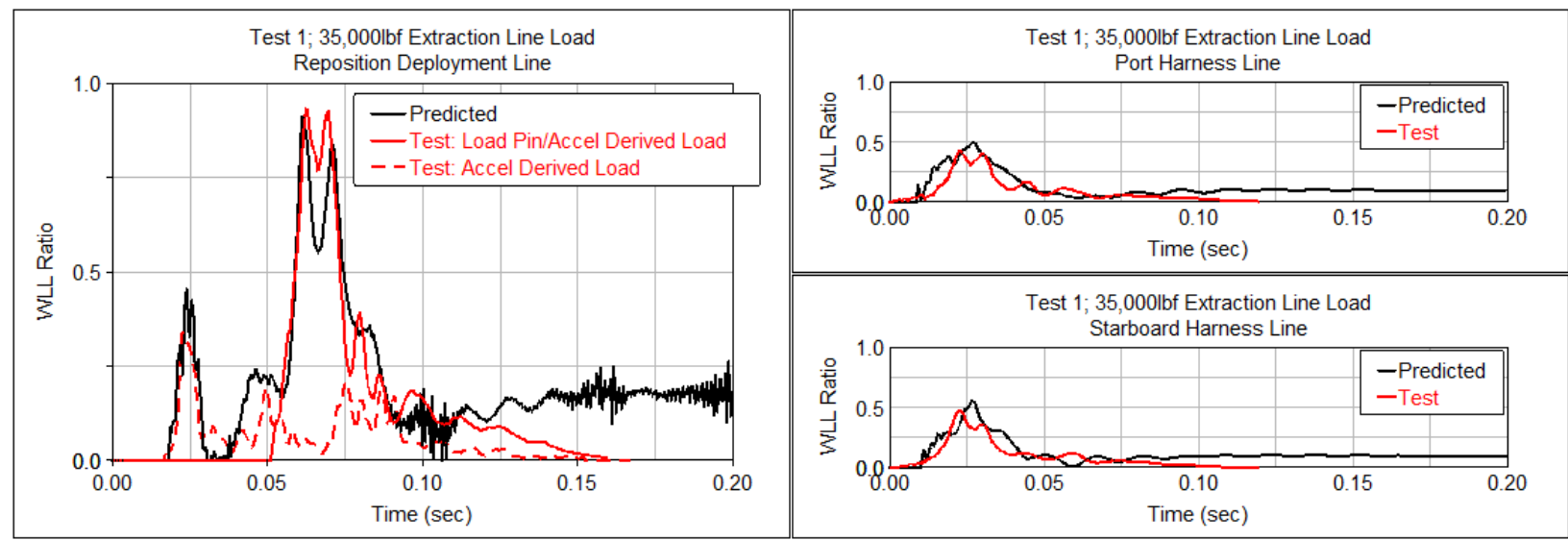

Figure 21. Test 1 reposition deployment and backstop harness line loads comparing the predicted value to test data

Completion of this dynamic ground testing has provided the CPAS project with confidence that the mitigation strategy of implementing a multi-loop energy modulator will provide the needed risk mitigation to continue flight testing from $35,000 \mathrm{ft}-\mathrm{MSL}$. As of the writing of this paper, the reconstruction effort is underway in order to reduce the difference between the predicted and as measured loads in order to provide analysis in support of the next CPAS drop test which is planned for the summer of 2017. 


\section{Conclusion}

The characterization of the $20 \mathrm{~K}$ Kevlar and type 26 nylon textile lines used during the various phases of a CPAS drop test has been challenging as well as insightful. Initial estimates were sufficient, however, with more complex and demanding drop tests the need to move from a simple linear model to a model based on static testing provided better line load estimation. A close call required additional testing a mitigation strategy that holds promise, exhibited in a dynamic environment, and will be implemented in the next drop test; ultimately allow testing once again from $35,000 \mathrm{ft}-\mathrm{MSL}$.

\section{Acknowledgments}

The authors of this paper would like to acknowledge Vladimir Drozd of Airborne Systems for his initial

recommendation for dynamic ground testing. As well as Calvin Flowers, Kyle Macy, Dave Richards and Bob of Holloway Houston, Inc. for their professionalism and expertise in executing both static and dynamic ground testing.

\section{References}

1 Ray, E.S., Varela, J.G., "Inflation of Unreefed and Reefed Extraction Parachutes," 23 ${ }^{\text {rd }}$ AIAA Aerodynamics Decelerator Systems Technology Conference, Daytona, FL, March 2015, AIAA Paper 2015-2180.

${ }^{2}$ Fraire, U., Anderson, K, Varela, J. and Bernatovich, M., "Extraction and Separation Modeling of Orion Test Vehicles with ADAMS Simulation: $2^{\text {nd }}$ Edition", 23 ${ }^{\text {rd }} A I A A$ Aerodynamic Decelerator Systems Technology Conference, Daytona, FL, March 2015, AIAA Paper 2015-2161. 